\title{
EFEITOS DAS CONDIÇÕES AMBIENTAIS SOBRE O TEOR E VARIABILIDADE DOS ÓLEOS VOLÁTEIS DE Dalbergia frutescens (Vell.) Britton (Fabaceae)
}

Caroline Eliza Mendes*, Fabiana Casarin e Anderson Luis Ohland

Área de Ciências Exatas e Ambientais, Universidade Comunitária da Região de Chapecó, Av. Senador Atílio Fontana, 591E, 89809-000 Chapecó - SC, Brasil

Adriana Flach e Luiz Antonio Mendonça Alves da Costa

Departamento de Química, Universidade Federal de Roraima, Av. Cap. Enê Garcêz, 2413, B1. III, 69304-000 Boa Vista - RR, Brasil Rosiane Berenice Nicoloso Denardin

Curso de Agronomia, Universidade Federal da Fronteira Sul, Av. Pres. Getúlio Vargas, 609N, 89812-000 Chapecó - SC, Brasil Neusa Fernandes de Moura

Escola de Química e Alimentos, Universidade Federal do Rio Grande, Rua Barão do Caí, 125, 955000-000 Santo Antonio da Patrulha - RS, Brasil

Recebido em 8/2/12; aceito em 11/6/12; publicado na web em 14/8/12

\begin{abstract}
EFFECTS OF ENVIRONMENTAL CONDITIONS ON THE CONTENT AND CHEMICAL VARIABILITY OF VOLATILE OILS OF Dalbergia frutescens (Vell.) Britton (Fabaceae). The volatile oils of Dalbergia frutescens were obtained by hydrodistillation on a Clevenger-modified apparatus for every month of one year and assessed on GC/MS and GC/FID detectors for qualitative and quantitative analyses. The influence on content of volatile oils was directly proportional to the environmental variables, temperature and cloudiness, and inversely proportional to precipitation. Among the volatile compounds detected, linalool, $\beta$-damascenone, $\alpha$-ionone, geranyl acetone and $\beta$-ionone were the main components, of which $\beta$-damascenone and $\beta$-ionone were found at the highest concentrations.
\end{abstract}

Keywords: Dalbergia frutescens; seasonality; variation volatile compounds.

\section{INTRODUÇÃO}

A constante descoberta de compostos bioativos a partir de produtos naturais, aliada à contínua ameaça à biodiversidade pela destruição dos ecossistemas terrestre e marinho justifica, irrefutavelmente, a colaboração multidisciplinar e internacional para o conhecimento e a exploração da natureza como fonte para o desenvolvimento de novos agentes bioativos. ${ }^{1}$

Devido à sua presença frequente nas espécies vegetais, facilidade de acesso e variedade de composição química, os óleos vêm sendo objetos de extensivos estudos visando a identificação de atividades biológicas, cujos resultados apontam para um potencial terapêutico importante. $^{2}$

Na dinâmica de crescimento, desenvolvimento e estádios fenológicos das espécies vegetais, há alterações bioquímicas e fisiológicas capazes de modificar a elaboração de substâncias biologicamente ativas, influenciando diretamente no teor e na qualidade dos óleos essenciais (fixos ou voláteis). ${ }^{3}$ Por esse motivo, os óleos não são constantes em termos qualitativos e quantitativos, sendo diretamente influenciados por fatores ambientais como nutrição, luminosidade, temperatura, radiação, déficit hídrico, local e horário de coleta. ${ }^{4}$

O gênero Dalbergia (Fabaceae) compreende cerca de 100 espécies de árvores, arbustos e lianas (cipós e trepadeiras), sendo que no Brasil ocorrem 40 espécies deste gênero, as quais são distribuídas em praticamente todos os ecossistemas, destacando-se as vegetações de Caatinga, Cerrado, Mata Atlântica e Campo Rupestre. ${ }^{5}$

Muitas espécies de Dalbergia são economicamente importantes devido a sua valiosa madeira, entretanto, a destruição acelerada das florestas tropicais, a fragmentação de habitats e a superexploração extrativista têm inserido muitas destas espécies de interesse econômico

*e-mail: carol_engquimica@yahoo.com.br e conservacionista no grupo de espécies ameaçadas de extinção, como o que vem ocorrendo com D. nigra (Jacarandá-da-Bahia). ${ }^{6}$

Nos últimos anos o gênero Dalbergia vem se destacando pelas inúmeras atividades biológicas detectadas em suas espécies, que incluem atividade anti-inflamatória, antirreumática, antioxidante, antibacteriana $^{7}$ e outras.

A espécie Dalbergia frutescens (sinonímia D. variabilis e Pterocarpus frutescens) é conhecida popularmente como Jacarandábranco, Cipó-pau, Cipó-de-estribo, Rabo-de-Bugio e Canela-doBrejo. ${ }^{9}$ A princípio, um dos únicos relatos de estudo de composição química para desta espécie foi realizado por Khan et al., ${ }^{8}$ no qual foram isoladas algumas isoflavonas, entre elas a formononetina, que demonstrou forte atividade antiprotozoárica contra Giardia intestinalis.

Tendo como base os estudos terapêuticos e químicos elencados anteriormente para o gênero Dalbergia, percebe-se um potencial interessante por parte de $D$. frutescens, embora ainda pouco explorado. Desta forma, como até o presente momento não há relatos de estudo de óleos voláteis desta espécie, torna-se iminente a necessidade de tal estudo que irá contribuir na identificação do potencial químico da biodiversidade da região oeste de Santa Catarina. Portanto, o objetivo do presente trabalho consistiu na avaliação sazonal do teor e da composição química dos óleos voláteis presentes em $D$. frutescens, contrapondo tais dados com as condições ambientais do período avaliado.

\section{RESULTADOS E DISCUSSÃO}

\section{Influência das condições ambientais sobre o rendimento}

O teor de óleos voláteis das folhas de D. frutescens variou de forma significativa ao longo do ano, atingindo o valor máximo de 
0,0076\% em janeiro e mínimo de 0,0019\% em agosto. A influência dos fatores climáticos sobre o rendimento foi avaliada através da análise de regressão linear múltipla, relacionando o teor de óleos voláteis com os valores médios de temperatura média, precipitação, insolação e nebulosidade (Tabela 1). Os coeficientes estatísticos obtidos a partir desta análise estão expressos na Tabela 2.

Pela análise de regressão linear múltipla, verificou-se que dos fatores abióticos avaliados, somente a variável insolação não influenciou significativamente o teor de óleos voláteis das folhas de $D$. frutescens. Das variáveis significativas, a temperatura e a nebulosidade influenciaram de forma diretamente proporcional a variável resposta. Entretanto, o inverso é observado para a precipitação, a qual influencia de forma inversamente proporcional o rendimento de óleo volátil. $\mathrm{O}$ alto valor do coeficiente Beta (Tabela 2) para a variável temperatura indica uma forte influência desta variável sobre o rendimento.

Segundo Ribeiro et al. ${ }^{10}$ a temperatura é um dos mais importantes fatores ambientais que influenciam a fisiologia das plantas, sendo a atividade fotossintética especialmente sensível a mudanças de temperatura. A fotossíntese, não somente determina o crescimento e desenvolvimento das plantas através da incorporação de carbono, mas também consiste no passo inicial para a síntese de metabólitos secundários, incluindo os óleos essenciais.

Para plantas do tipo $\mathrm{C}_{3}$ como é o caso de $D$. frutescens, a fotossíntese atinge seu auge em temperaturas variando de 25 a 30 ${ }^{\circ} \mathrm{C}$, desta forma, nos meses frios haverá uma redução da atividade fotossintética e de processos metabólicos. Aliado a este fato, a síntese de terpenoides confere estabilidade às membranas fotossintéticas, quando as folhas são submetidas à temperatura e luminosidade altas, o que torna a síntese de terpenoides proporcional à temperatura da folha e ao estresse hídrico. ${ }^{11}$ Segundo Fitter e Hay, ${ }^{12}$ em meses quentes com baixa precipitação e umidade relativa, as plantas acionam seu mecanismo de defesa contra a perda de água, fechando seus estômatos e produzindo óleos essenciais. Segundo Vitti e Brito, ${ }^{13}$ os meses mais secos do ano propiciam um maior rendimento de óleo essencial, fato semelhante ao que ocorreu neste e em outros estudos. ${ }^{14-16}$

\section{Influência das condições ambientais sobre a composição química}

Foram detectados nas folhas de $D$. frutescens um total de 60 compostos presentes no óleo volátil, correspondendo a uma média anual de 93,63\% de compostos identificados, destacando-se a classe dos norisoprenoides e dos sesquiterpenos. A Tabela 3 apresenta a variação anual dos compostos identificados na $D$. frutescens.

A classe dos norisoprenoides prevaleceu em praticamente todos os meses do ano, em concentrações que variaram de $11,5 \%$, em junho, a $60,5 \%$ em dezembro, mantendo uma média anual de aproximadamente $39 \%$, a maior média verificada para as classes de compostos identificados. De maneira geral, não foi observada semelhança nas variações das condições ambientais com as concentrações encontradas para estes compostos ao longo do período avaliado.

Os hidrocarbonetos sesquiterpênicos aparentemente não sofreram influência das variáveis ambientais, apesar de em abril, mês que apresentou a maior concentração de compostos desta classe de metabólitos $(29,45 \%)$, ser registrada a maior precipitação do período. Exceto este ponto, os demais não se comportaram de forma coincidente às variações ambientais.

Tabela 1. Rendimento percentual do óleo volátil de $D$. frutescens e valores médios de temperatura, precipitação, insolação e nebulosidade, mensalmente ao longo de um ano

\begin{tabular}{|c|c|c|c|c|c|}
\hline Mês/ano & Rendimento $[\%]^{1}$ & Temperatura média $\left[{ }^{\circ} \mathrm{C}\right]^{2}$ & Precipitação $[\mathrm{mm}]^{2}$ & Insolação $[\mathrm{h}]^{2}$ & Nebulosidade $[1 / 10]^{2}$ \\
\hline $01 / 2011$ & $0,0076^{g}$ & 24,71 & 82,0 & 98,1 & 6,58 \\
\hline $02 / 2011$ & $0,0060^{\mathrm{f}}$ & 23,54 & 121,0 & 73,5 & 6,87 \\
\hline 03/2010 & $0,0044^{\mathrm{d}}$ & 22,00 & 159,9 & 57,5 & 7,05 \\
\hline $04 / 2010$ & $0,0038^{\mathrm{c}}$ & 20,21 & 183,8 & 51,3 & 6,90 \\
\hline $05 / 2010$ & $0,0032^{\mathrm{c}}$ & 15,61 & 107,3 & 83,9 & 6,50 \\
\hline $06 / 2010$ & $0,0024^{\mathrm{b}}$ & 13,65 & 114,2 & 72,0 & 6,31 \\
\hline $07 / 2010$ & $0,0035^{\mathrm{c}}$ & 15,39 & 102,0 & 82,0 & 6,63 \\
\hline $08 / 2010$ & $0,0019^{\mathrm{a}}$ & 12,68 & 95,4 & 57,8 & 5,30 \\
\hline $09 / 2010$ & $0,0025^{\mathrm{b}}$ & 17,54 & 104,9 & 86,6 & 5,74 \\
\hline $10 / 2010$ & $0,0039^{\mathrm{c}}$ & 19,61 & 69,2 & 102,2 & 4,53 \\
\hline $11 / 2010$ & $0,0046^{\mathrm{d}}$ & 22,04 & 75,1 & 128,7 & 5,23 \\
\hline $12 / 2010$ & $0,0056^{\mathrm{e}}$ & 23,30 & 23,5 & 121,9 & 4,80 \\
\hline
\end{tabular}

${ }^{1}$ Médias seguidas de letra minúscula diferente entre linhas diferem significativamente entre si pelo teste de Tukey a $5 \%$ de probabilidade. ${ }^{2}$ Valores médios da semana anterior à coleta. Dados disponibilizados pela Empresa de Pesquisa Agropecuária e Extensão Rural de Santa Catarina - EPAGRI de Chapecó-SC.

Tabela 2. Coeficientes estatísticos obtidos por regressão linear múltipla para a influência das variáveis temperatura média, precipitação, insolação e nebulosidade sobre o rendimento do óleo volátil foliar de Dalbergia frutescens

\begin{tabular}{|c|c|c|c|c|c|c|}
\hline & $\mathrm{R}^{2}$ ajustado & $\mathrm{F}(4,7)$ & Erro quadrático estimado & Beta & B & Nível p \\
\hline Modelo & 0,9206 & 32,885 & 0,00047 & - & - & 0,00013 \\
\hline Intercept & & & & - & $-0,004481$ & 0,049688 \\
\hline Temperatura $\left[{ }^{\circ} \mathrm{C}\right]$ & & & & 0,8738 & 0,000358 & 0,000081 \\
\hline Precipitação [mm] & & & & $-0,7253$ & $-0,000029$ & 0,007632 \\
\hline Insolação [h] & & & & $-0,2340$ & $-0,000016$ & 0,255272 \\
\hline Nebulosidade [1/10] & & & & 0,5309 & 0,001019 & 0,006997 \\
\hline
\end{tabular}


Tabela 3. Composição química do óleo volátil de Dalbergia frutescens mensalmente, ao longo de um ano

\begin{tabular}{|c|c|c|c|c|c|c|c|c|c|c|c|c|c|c|c|}
\hline \multirow[t]{2}{*}{ Composto } & \multirow[t]{2}{*}{ Classe } & \multicolumn{2}{|c|}{$\begin{array}{l}\text { Índice de } \\
\text { Retenção }\end{array}$} & \multicolumn{12}{|c|}{ Concentração $[\%]^{3}$} \\
\hline & & $\mathrm{IRL}_{\mathrm{C}}{ }^{1}$ & $\mathrm{IRL}_{\mathrm{L}}^{2}$ & JAN & FEV & MAR & $\mathrm{ABR}$ & MAI & JUN & JUL & AGO & SET & OUT & $\mathrm{NOV}$ & DEZ \\
\hline (E)-2-hexenal & ALD & 859 & 855 & n.d. & n.d. & n.d. & n.d. & $1,89 \mathrm{~b}$ & n.d. & $5,57 \mathrm{c}$ & n.d. & n.d. & n.d. & n.d. & n.d. \\
\hline (Z)-3-hexenol & ALC & 861 & 859 & n.d. & n.d. & n.d. & n.d. & $11,73 \mathrm{c}$ & $21,4 d$ & $7,69 b$ & n.d. & n.d. & n.d. & n.d. & n.d. \\
\hline n-hexanol & ALC & 871 & 871 & n.d. & n.d. & n.d. & n.d. & $30,74 \mathrm{c}$ & $51,31 d$ & $9,54 \mathrm{~b}$ & n.d. & n.d. & n.d. & n.d. & n.d. \\
\hline$(\mathrm{E}, \mathrm{E})-2,4$-hexadienal & HD & 910 & 910 & n.d. & $0,86 b$ & n.d. & n.d. & n.d. & n.d. & n.d. & n.d. & n.d. & n.d. & n.d. & n.d. \\
\hline Metanoato de hexila & EST & 932 & 929 & n.d. & $0,18 \mathrm{a}$ & n.d. & n.d. & n.d. & n.d. & n.d. & n.d. & n.d. & n.d. & n.d. & n.d. \\
\hline$\alpha$-tujeno & $\mathrm{MH}$ & 934 & 930 & n.d. & n.d. & n.d. & n.d. & n.d. & n.d. & n.d. & n.d. & n.d. & n.d. & n.d. & $0,72 b$ \\
\hline Heptanol & $\mathrm{ALC}$ & 970 & 967 & n.d. & n.d. & $0,46 b$ & n.d. & n.d. & n.d. & n.d. & n.d. & n.d. & n.d. & n.d. & n.d. \\
\hline 1-octen-3-ol & ALC & 979 & 979 & $0,50 \mathrm{~b}$ & $1,06 \mathrm{c}$ & $1,51 \mathrm{~d}$ & n.d. & $0,57 b$ & n.d. & n.d. & n.d. & n.d. & n.d. & n.d. & n.d. \\
\hline (E,E)-2,4-heptadienal & HD & 1016 & 1013 & n.d. & $0,28 b$ & n.d. & n.d. & n.d. & n.d. & $1,00 \mathrm{c}$ & n.d. & n.d. & n.d. & n.d. & n.d. \\
\hline Limoneno & $\mathrm{MH}$ & 1029 & 1029 & n.d. & n.d. & n.d. & n.d. & n.d. & n.d. & n.d. & n.d. & n.d. & n.d. & n.d. & $2,89 b$ \\
\hline Fenil-acetaldeído & ALD & 1042 & 1042 & n.d. & $0,36 b$ & n.d. & n.d. & n.d. & n.d. & n.d. & n.d. & n.d. & n.d. & n.d. & n.d. \\
\hline (E)- $\beta$-ocimeno & $\mathrm{MH}$ & 1048 & 1050 & n.d. & $0,53 \mathrm{~b}$ & n.d. & n.d. & n.d. & n.d. & n.d. & n.d. & n.d. & n.d. & n.d. & n.d. \\
\hline Octanol & ALC & 1071 & 1068 & n.d. & n.d. & n.d. & n.d. & n.d. & n.d. & n.d. & n.d. & $0,66 \mathrm{~b}$ & n.d. & n.d. & n.d. \\
\hline$<$ Trans>-óxido de linalol & MO & 1073 & 1073 & n.d. & $0,27 \mathrm{~b}$ & n.d. & n.d. & n.d. & n.d. & n.d. & n.d. & $0,74 \mathrm{c}$ & n.d. & n.d. & n.d. \\
\hline Linalol & $\mathrm{MO}$ & 1100 & 1097 & $8,47 \mathrm{f}$ & $8,63 \mathrm{~g}$ & $10,43 \mathrm{~h}$ & n.d. & $3,54 b$ & $4,65 d$ & $4,72 d$ & $3,79 \mathrm{c}$ & $6,07 \mathrm{e}$ & $12,43 \mathrm{i}$ & $14,71 \mathrm{k}$ & $13,76 \mathrm{j}$ \\
\hline Nonanal & ALD & 1103 & 1101 & $0,54 b$ & $1,11 \mathrm{~cd}$ & $1,28 \mathrm{~d}$ & n.d. & n.d. & n.d. & n.d. & n.d. & $1,65 \mathrm{e}$ & n.d. & $1,06 \mathrm{c}$ & n.d. \\
\hline (E)-2,6-dimetil-1,3,7-octatrien-6-ol & MO & 1112 & 1101 & n.d. & n.d. & n.d. & n.d. & n.d. & n.d. & $1,39 \mathrm{~b}$ & n.d. & n.d. & n.d. & n.d. & n.d. \\
\hline $\begin{array}{l}\text { 2-metil-6-metileno-1,7-octadien-3- } \\
\text {-ona }\end{array}$ & MO & 1119 & 1117 & n.d. & $0,68 \mathrm{~b}$ & n.d. & n.d. & n.d. & n.d. & n.d. & n.d. & n.d. & n.d. & n.d. & n.d. \\
\hline$\alpha$-terpineol & MO & 1190 & 1189 & $0,83 \mathrm{~cd}$ & $0,99 d$ & $0,50 \mathrm{~b}$ & n.d. & n.d. & n.d. & $0,64 b c$ & n.d. & n.d. & n.d. & $1,30 \mathrm{e}$ & $1,44 \mathrm{e}$ \\
\hline Safranal & MO & 1201 & 1201 & n.d. & n.d. & n.d. & n.d. & n.d. & n.d. & $0,75 b$ & n.d. & n.d. & n.d. & n.d. & n.d. \\
\hline Beta-ciclo-citral & MO & 1219 & 1219 & n.d. & $0,79 b$ & n.d. & n.d. & n.d. & n.d. & n.d. & n.d. & n.d. & n.d. & n.d. & n.d. \\
\hline$<$ Cis, para $>$ menta-1(7)-,8-dien-2-ol & MO & 1234 & 1231 & $0,70 \mathrm{c}$ & n.d. & $0,44 b$ & n.d. & n.d. & n.d. & $0,81 \mathrm{c}$ & n.d. & n.d. & n.d. & n.d. & $1,45 \mathrm{~d}$ \\
\hline Pulegona & MO & 1242 & 1241 & n.d. & n.d. & n.d. & n.d. & n.d. & $6,87 \mathrm{c}$ & n.d. & n.d. & $1,09 b$ & n.d. & n.d. & n.d. \\
\hline Geraniol & MO & 1256 & 1255 & n.d. & $0,86 b$ & n.d. & n.d. & n.d. & n.d. & n.d. & n.d. & n.d. & n.d. & n.d. & n.d. \\
\hline 4-vinil-guaiacol & ALC & 1313 & 1309 & n.d. & n.d. & n.d. & n.d. & $2,76 \mathrm{c}$ & n.d. & $2,16 \mathrm{~b}$ & n.d. & n.d. & n.d. & n.d. & n.d. \\
\hline Acetato de citronelila & EST & 1340 & 1353 & n.d. & n.d. & n.d. & n.d. & n.d. & n.d. & n.d. & $1,87 \mathrm{~b}$ & n.d. & n.d. & n.d. & n.d. \\
\hline Acetato de verbanol & EST & 1349 & 1344 & n.d. & n.d. & n.d. & n.d. & n.d. & n.d. & $1,28 b$ & n.d. & n.d. & n.d. & n.d. & n.d. \\
\hline 2-butil-2-octenal & ALD & 1376 & 1378 & n.d. & n.d. & n.d. & n.d. & n.d. & n.d. & n.d. & n.d. & $1,75 b$ & n.d. & n.d. & n.d. \\
\hline$\alpha$-copaeno & $\mathrm{SH}$ & 1377 & 1377 & $1,84 \mathrm{f}$ & $0,82 \mathrm{~cd}$ & $0,41 \mathrm{~b}$ & n.d. & $5,02 \mathrm{~g}$ & n.d. & n.d. & n.d. & $0,93 \mathrm{de}$ & n.d. & $0,65 \mathrm{c}$ & $1,10 \mathrm{e}$ \\
\hline Caproato de 3(z)-hexenila & EST & 1383 & 1382 & $0,93 \mathrm{c}$ & n.d. & n.d. & n.d. & n.d. & n.d. & n.d. & n.d. & n.d. & n.d. & $0,55 b$ & n.d. \\
\hline (E)- $\beta$-damascenona & NOR & 1385 & 1385 & $15,83 f$ & $10,98 c$ & 29,721 & $21,70 \mathrm{j}$ & $16,26 \mathrm{~g}$ & $11,48 d$ & $23,58 \mathrm{k}$ & $18,38 \mathrm{~h}$ & n.d. & $4,50 \mathrm{~b}$ & $13,59 \mathrm{e}$ & $21,39 \mathrm{i}$ \\
\hline$\beta$-cubebeno & $\mathrm{SH}$ & 1390 & 1390 & $2,43 \mathrm{~d}$ & $1,11 \mathrm{~b}$ & $2,67 \mathrm{e}$ & $6,19 \mathrm{~g}$ & $1,63 \mathrm{c}$ & n.d. & n.d. & n.d. & n.d. & n.d. & n.d. & $3,91 \mathrm{f}$ \\
\hline$\beta$-longipineno & $\mathrm{SH}$ & 1402 & 1401 & n.d. & n.d. & n.d. & n.d. & n.d. & n.d. & $4,07 \mathrm{~b}$ & $5,29 \mathrm{c}$ & n.d. & n.d. & n.d. & n.d. \\
\hline$\alpha$-cidreno & $\mathrm{SH}$ & 1415 & 1412 & $1,03 \mathrm{~b}$ & $1,57 \mathrm{c}$ & $5,33 d$ & $23,26 \mathrm{~h}$ & $7,70 \mathrm{e}$ & n.d. & $14,08 \mathrm{~g}$ & $11,37 f$ & n.d. & n.d. & n.d. & n.d. \\
\hline (E)-cariofileno & $\mathrm{SH}$ & 1419 & 1419 & $1,76 \mathrm{c}$ & $0,92 b$ & n.d. & n.d. & n.d. & n.d. & n.d. & n.d. & n.d. & n.d. & n.d. & n.d. \\
\hline (E)- $\alpha$-ionona & NOR & 1430 & 1430 & $6,47 \mathrm{j}$ & $3,38 \mathrm{~g}$ & $5,88 \mathrm{i}$ & $2,86 \mathrm{e}$ & $1,46 b$ & n.d. & $2,53 \mathrm{~d}$ & $3,19 f$ & $2,52 \mathrm{~d}$ & $2,23 \mathrm{c}$ & $2,46 \mathrm{~d}$ & $5,55 \mathrm{~h}$ \\
\hline Geranil-acetona & NOR & 1455 & 1455 & $3,00 \mathrm{i}$ & $0,98 \mathrm{c}$ & $2,41 \mathrm{~h}$ & $1,55 \mathrm{e}$ & $0,71 b$ & n.d. & $2,16 \mathrm{~g}$ & $4,35 \mathrm{j}$ & $1,44 d$ & n.d. & $1,77 \mathrm{f}$ & $4,67 \mathrm{k}$ \\
\hline $\begin{array}{l}\text { (E)-4-(2,3,6-trimetil-fenil)-3-buten-2- } \\
\text {-ona }\end{array}$ & NOR & 1488 & 1475 & n.d. & n.d. & n.d. & n.d. & n.d. & n.d. & n.d. & $1,78 b$ & n.d. & n.d. & n.d. & n.d. \\
\hline (E)- $\beta$-ionona & NOR & 1489 & 1489 & $21,84 \mathrm{j}$ & $6,37 b$ & $13,92 f$ & $19,51 \mathrm{~h}$ & $6,88 \mathrm{c}$ & n.d. & $7,87 \mathrm{~d}$ & $9,54 \mathrm{e}$ & $20,5 \mathrm{i}$ & 52,321 & $16,43 \mathrm{~g}$ & $25,76 \mathrm{k}$ \\
\hline$<10,11$-epoxy $>$ calameneno & $\mathrm{SO}$ & 1494 & 1492 & $0,66 b$ & n.d. & n.d. & n.d. & n.d. & n.d. & n.d. & n.d. & $1,42 \mathrm{c}$ & n.d. & n.d. & n.d. \\
\hline Cubebol & $\mathrm{SO}$ & 1521 & 1518 & n.d. & n.d. & n.d. & n.d. & n.d. & n.d. & n.d. & n.d. & $1,21 \mathrm{~b}$ & n.d. & n.d. & n.d. \\
\hline$\delta$-cadineno & $\mathrm{SH}$ & 1523 & 1523 & n.d. & $0,30 \mathrm{~b}$ & n.d. & n.d. & n.d. & n.d. & n.d. & n.d. & n.d. & n.d. & n.d. & n.d. \\
\hline$<$ Trans $>$-calameneno & $\mathrm{SH}$ & 1529 & 1529 & $0,96 b$ & n.d. & n.d. & n.d. & n.d. & n.d. & n.d. & n.d. & $1,73 \mathrm{c}$ & n.d. & n.d. & n.d. \\
\hline $\begin{array}{l}\text { (R)-5,6,7,7- } \alpha \text {-tetrahidro-4-4-7a- } \\
\text { trimetil-2(4h)-benzofuranona }\end{array}$ & FUR & 1531 & 1525 & n.d. & n.d. & n.d. & n.d. & n.d. & n.d. & n.d. & n.d. & $2,84 b$ & n.d. & n.d. & n.d. \\
\hline$\alpha$-calacoreno & $\mathrm{SH}$ & 1546 & 1546 & $3,57 \mathrm{f}$ & $0,36 \mathrm{~b}$ & $0,67 \mathrm{c}$ & n.d. & n.d. & n.d. & n.d. & n.d. & n.d. & n.d. & $1,57 \mathrm{e}$ & $1,25 \mathrm{~d}$ \\
\hline Epóxido italiceno & SO & 1552 & 1549 & n.d. & n.d. & n.d. & n.d. & n.d. & n.d. & n.d. & n.d. & $0,72 b$ & n.d. & n.d. & n.d. \\
\hline (E)-nerolidol & $\mathrm{SO}$ & 1564 & 1563 & $1,16 \mathrm{c}$ & $0,57 b$ & $0,44 b$ & n.d. & n.d. & n.d. & n.d. & $3,11 \mathrm{e}$ & $1,96 \mathrm{~d}$ & n.d. & n.d. & n.d. \\
\hline (3-z)-benzoato de hexenila & EST & 1573 & 1573 & $3,41 \mathrm{~d}$ & $0,55 \mathrm{~b}$ & $14,32 \mathrm{f}$ & n.d. & n.d. & n.d. & n.d. & $3,17 \mathrm{c}$ & n.d. & n.d. & $12,48 \mathrm{e}$ & n.d. \\
\hline Dendrolasin & $\mathrm{SO}$ & 1582 & 1582 & $2,06 \mathrm{c}$ & $50,84 f$ & $1,14 \mathrm{~b}$ & n.d. & n.d. & n.d. & n.d. & $8,87 \mathrm{~d}$ & n.d. & n.d. & $16,96 \mathrm{e}$ & n.d. \\
\hline Espatulenol & $\mathrm{SO}$ & 1585 & 1578 & $1,35 b$ & n.d. & n.d. & $10,79 f$ & $3,69 \mathrm{e}$ & n.d. & n.d. & $2,08 \mathrm{~d}$ & $1,74 \mathrm{c}$ & n.d. & n.d. & n.d. \\
\hline Óxido de cariofileno & $\mathrm{SO}$ & 1588 & 1587 & $1,27 \mathrm{~b}$ & n.d. & n.d. & $5,47 \mathrm{c}$ & n.d. & n.d. & n.d. & n.d. & $5,48 \mathrm{c}$ & n.d. & n.d. & n.d. \\
\hline$\beta$-acorenol & $\mathrm{SO}$ & 1636 & 1636 & n.d. & n.d. & n.d. & n.d. & n.d. & n.d. & n.d. & n.d. & $2,37 \mathrm{~b}$ & n.d. & n.d. & n.d. \\
\hline
\end{tabular}


Tabela 3. continuação

\begin{tabular}{|c|c|c|c|c|c|c|c|c|c|c|c|c|c|c|c|}
\hline \multirow[t]{2}{*}{ Composto } & \multirow[t]{2}{*}{ Classe } & \multicolumn{2}{|c|}{$\begin{array}{l}\text { Índice de } \\
\text { Retenção }\end{array}$} & \multicolumn{12}{|c|}{ Concentração $[\%]^{3}$} \\
\hline & & $\mathrm{IRL}_{\mathrm{C}}{ }^{1}$ & $\mathrm{IRL}_{\mathrm{L}}{ }^{2}$ & JAN & FEV & MAR & ABR & MAI & JUN & JUL & AGO & SET & OUT & NOV & DEZ \\
\hline Cubenol & $\mathrm{SO}$ & 1650 & 1647 & n.d. & n.d. & n.d. & n.d. & n.d. & n.d. & n.d. & n.d. & $1,44 \mathrm{~b}$ & n.d. & n.d. & n.d. \\
\hline$\alpha$-cadinol & SO & 1654 & 1654 & $4,23 \mathrm{~d}$ & $0,8 b$ & n.d. & $0,71 b$ & n.d. & n.d. & n.d. & n.d. & $8,22 \mathrm{f}$ & $7,45 \mathrm{e}$ & $3,87 \mathrm{c}$ & $4,04 \mathrm{c}$ \\
\hline Cadaleno & $\mathrm{SH}$ & 1678 & 1677 & $0,80 \mathrm{~b}$ & n.d. & n.d. & n.d. & n.d. & n.d. & n.d. & n.d. & $3,35 \mathrm{c}$ & n.d. & n.d. & n.d. \\
\hline Tetradecanol & ALC & 1681 & 1680 & n.d. & n.d. & n.d. & n.d. & n.d. & n.d. & $1,14 \mathrm{~b}$ & $3,23 \mathrm{c}$ & $6,86 \mathrm{~d}$ & n.d. & n.d. & n.d. \\
\hline Pentadecanal & SO & 1712 & 1711 & n.d. & n.d. & n.d. & n.d. & n.d. & n.d. & $2,71 \mathrm{c}$ & $3,68 \mathrm{~d}$ & n.d. & $1,39 \mathrm{~b}$ & n.d. & n.d. \\
\hline Pentadecan-2-ona & SO & 1843 & 1841 & $1,56 \mathrm{~d}$ & n.d. & $0,66 b$ & n.d. & n.d. & n.d. & $1,04 \mathrm{c}$ & $6,14 \mathrm{~g}$ & $6,54 \mathrm{~h}$ & $4,66 f$ & $1,90 \mathrm{e}$ & $1,64 \mathrm{~d}$ \\
\hline$(\mathrm{E}, \mathrm{E})$-farnesil acetona & NOR & 1921 & 1921 & $3,49 \mathrm{e}$ & $0,27 b$ & $1,01 \mathrm{c}$ & n.d. & n.d. & n.d. & n.d. & $3,52 \mathrm{e}$ & $0,92 \mathrm{c}$ & n.d. & $0,99 \mathrm{c}$ & $3,19 \mathrm{~d}$ \\
\hline Hexadecanoato de metila & EST & 1925 & 1925 & n.d. & n.d. & n.d. & n.d. & n.d. & n.d. & n.d. & n.d. & $9,78 \mathrm{c}$ & $10,32 \mathrm{~d}$ & $0,58 \mathrm{~b}$ & n.d. \\
\hline TOTAL & & & & 90,69 & 96,42 & 93,20 & 92,04 & 94,58 & 95,71 & 94,73 & 93,36 & 93,93 & $\mathbf{9 5 , 3}$ & 90,87 & 92,76 \\
\hline ALD: Aldeídos & & & & 0,54 & 1,47 & 1,28 & 0,00 & 1,89 & 0,00 & 5,57 & 0,00 & 3,40 & 0,00 & 1,06 & 0,00 \\
\hline ALC: Alcoóis & & & & 0,50 & 1,06 & 1,97 & 0,00 & 45,8 & 72,71 & 20,53 & 3,23 & 7,52 & 0,00 & 0,00 & 0,00 \\
\hline HD: Hidrocarbonetos & & & & 0,00 & 1,14 & 0,00 & 0,00 & 0,00 & 0,00 & 1,00 & 0,00 & 0,00 & 0,00 & 0,00 & 0,00 \\
\hline NOR: Norisoprenoides & & & & 50,63 & 21,98 & 52,94 & 45,62 & 25,31 & 11,48 & 36,14 & 40,76 & 25,38 & 59,05 & 35,24 & 60,56 \\
\hline FUR: Furanonas & & & & 0,00 & 0,00 & 0,00 & 0,00 & 0,00 & 0,00 & 0,00 & 0,00 & 2,84 & 0,00 & 0,00 & 0,00 \\
\hline EST: Ésteres & & & & 4,34 & 0,73 & 14,32 & 0,00 & 0,00 & 0,00 & 1,28 & 5,04 & 9,78 & 10,32 & 13,61 & 0,00 \\
\hline $\begin{array}{l}\text { MH: Hidrocarbonetos monoterpê- } \\
\text { nicos }\end{array}$ & & & & 0,00 & 0,53 & 0,00 & 0,00 & 0,00 & 0,00 & 0,00 & 0,00 & 0,00 & 0,00 & 0,00 & 3,61 \\
\hline MO: Monoterpenos oxigenados & & & & 10,00 & 12,22 & 11,37 & 0,00 & 3,54 & 11,52 & 8,31 & 3,79 & 7,90 & 12,43 & 16,01 & 16,65 \\
\hline $\begin{array}{l}\text { SH: Hidrocarbonetos sesquiterpê- } \\
\text { nicos }\end{array}$ & & & & 12,39 & 5,08 & 9,08 & 29,45 & 14,35 & 0,00 & 18,15 & 16,66 & 6,01 & 0,00 & 2,22 & 6,26 \\
\hline SO: Sesquiterpenos oxigenados & & & & 12,29 & 52,21 & 2,24 & 16,97 & 3,69 & 0,00 & 3,75 & 23,88 & 31,10 & 13,50 & 22,73 & 5,68 \\
\hline
\end{tabular}

${ }^{1}$ Índice de Retenção Linear calculado com base em uma mistura padrão de hidrocarbonetos C7-C30. ${ }^{2}$ Índice de Retenção Linear da literatura, obtido para coluna RTX-5 em ADAMS. ${ }^{263}$ Concentração percentual calculada com base nas áreas dos picos, sendo os valores médios de três injeções. Médias seguidas de letra diferente entre colunas, diferem significativamente entre si pelo teste de Tukey a 5\% de probabilidade. n.d. - Não detectado pelo método empregado.

Os monoterpenos oxigenados mantiveram uma média anual de aproximadamente $9,47 \%$, com o teor mínimo ocorrido em abril e máximo em dezembro. Contrapondo estes dados com os de precipitação (Tabela 1), pode-se observar que a alta precipitação ocorrida no mês de abril $(183,8 \mathrm{~mm})$ pode ter contribuído para o valor mínimo observado, da mesma forma que a baixa precipitação de dezembro $(23,5 \mathrm{~mm})$ aparentemente maximizou a concentração de monoterpenos oxigenados.

A concentração de alcoóis, entretanto, aparenta estar diretamente relacionada à temperatura média do ambiente, havendo maiores concentrações de alcoóis em meses frios como maio, junho e julho.

Conforme a Tabela 3, alguns compostos químicos apresentaram-se de forma bastante característica, sendo detectados em no mínimo 10 meses do ano nas folhas de D. frutescens, como é o caso do linalol (IRL 1097), $\beta$-damascenona (IRL 1385), $\alpha$-ionona (IRL 1430), geranil-acetona (IRL 1455) e $\beta$-ionona (IRL 1489).

Observa-se que o linalol somente não esteve presente na composição química da espécie no mês de abril, fato que pode estar relacionado à alta precipitação ocorrida nesse mês, que foi a maior registrada para o período analisado (Tabela 1). Pode-se afirmar ainda que este composto está presente em maiores concentrações nos meses que apresentaram os menores índices de precipitação anual (23,50 a 75,10 mm) e maiores níveis de insolação (102,20 a $128,70 \mathrm{~h}$ ), como outubro, novembro e dezembro, os quais apresentaram concentração percentual deste composto superior a 12\%. Já a concentração da $\alpha$-ionona e da geranil-acetona em todos os meses não ultrapassou $10 \%$, sendo a variação sazonal destes compostos bastante semelhante.

Entre os compostos de maior concentração destacam-se $\beta$-damascenona e $\beta$-ionona. $O$ primeiro apresentou concentrações maiores do que a $\beta$-ionona nos meses que compõem as estações de outono e inverno, enquanto a $\beta$-ionona se sobressaiu nos meses das estações de primavera e verão.
A $\beta$-damascenona foi detectada em praticamente todos os meses, não sendo observada como constituinte majoritário somente nos meses de setembro e outubro. Sua concentração variou de $10,98 \%$ (fevereiro) a 29,72\% (março), oscilando consideravelmente em todos os meses. Altas concentrações de $\beta$-ionona foram detectadas de setembro a janeiro, onde especificamente no mês de setembro, este composto foi o único constituinte majoritário do óleo volátil, em uma concentração de $20,5 \%$. Seu pico máximo de concentração foi constatado em outubro, chegando a $52,32 \%$, o que pode estar relacionado com os estádios fenológicos desta espécie, tendo em vista que nos meses de setembro e outubro há uma aceleração do metabolismo vegetal, gerando um grande número de folhas novas. No término do outono e inverno pode-se observar que este constituinte não se apresentou como majoritário, época em que as folhas em senescência são predominantes.

Segundo Baldermann et al. ${ }^{17}$ os constituintes $\alpha$-ionona e $\beta$-ionona são produzidos pela degradação de $\alpha$-caroteno, o que justifica seu comportamento semelhante ao longo do ano. Entretanto, observa-se que o teor de $\beta$-ionona foi consideravelmente superior ao de $\alpha$-ionona, fato que pode ser justificado por uma degradação conjunta de $\alpha$-caroteno e $\beta$-caroteno. Conforme Simkin et al. ${ }^{18}$ ao ocorrer a clivagem oxidativa do $\beta$-caroteno, são formadas duas moléculas de $\beta$-ionona havendo, portanto, uma alta produção deste constituinte. Caso ambas as moléculas, $\alpha$-caroteno e $\beta$-caroteno, sejam clivadas, o que se acredita que ocorreu neste estudo, haveria uma produção três vezes superior de $\beta$-ionona em comparação à de $\alpha$-ionona. Além disso, segundo Yamamizo et al., ${ }^{19}$ a neoxantina, xantofila que dá origem à $\beta$-damascenona, é obtida a partir do $\beta$-caroteno, o que justifica a ocorrência de níveis máximos de $\beta$-damascenona e $\beta$-ionona em estádios fenológicos distintos.

Alguns dos compostos detectados não ocorreram em todos os meses avaliados, entretanto, muitos foram encontrados em épocas do ano bastante específicas. Este é o caso de alcoóis simples, como 
o 3-hexenol (IRL 859) e o n-hexanol (IRL 871), os quais foram detectados somente em meses frios (maio, junho e julho). Tendo em vista que sua época de ocorrência se limitou ao estádio fenológico em que as folhas iniciam sua senescência, a detecção de concentrações altas destes constituintes pode estar vinculada à degradação enzimática de ácidos graxos insaturados, reação que produz compostos como aldeídos, cetonas e alcoóis de baixo peso molecular, conforme relatado por Hatanaka. ${ }^{20}$

Observa-se ainda que em determinados meses, como fevereiro e setembro, foram detectados inúmeros compostos com baixa concentração, fato que pode estar relacionado ao mecanismo de defesa ou atração de polinizadores de forma sinérgica. Segundo Fernandes, ${ }^{21}$ a espécie Dalbergia frutescens floresce entre novembro e março. Pelas observações realizadas em campo, a floração da matriz utilizada ocorreu em fevereiro, havendo neste mês a ocorrência dos compostos 2,4-hexadienal (IRL 910), metanoato de hexila (IRL 929), fenil-acetaldeído (IRL 1042), $\beta$-ocimeno (IRL 1050), 2-metil-6-metileno-1,7-octadien-3-ona (IRL 1117), $\beta$-ciclo-citral (IRL 1219), geraniol (IRL 1255), $\delta$-cadineno (IRL 1523) em quantidades inferiores a $1 \%$. Compostos como o geraniol e o 2-metil-6-metileno-1,7-octadien-3-ona são relatados como feromônios de abelhas, ${ }^{22,23}$ assim como há relatos da produção de $\beta$-ocimeno pelas flores das plantas para atração de polinizadores. ${ }^{24}$

Em setembro, mês em que o número de folhas novas estava no auge, observou-se o aparecimento de compostos como octanol (IRL 1068), 2-butil-2-octenal (IRL 1378), epóxido italiceno (IRL 1549), cubebol (IRL 1518), $\beta$-acorenol (IRL 1636) e cubenol (IRL 1647) em concentrações variando de 0,66 a 2,37\%. O dendrolasin (IRL 1582) pode ser observado como constituinte majoritário no mês de fevereiro (50,84\%), o qual dificilmente é encontrado como composto volátil de plantas, sendo detectado com maior ocorrência como um sistema de defesa contra formigas; entretanto, em estudos com óleo essencial de folhas e flores de Brugmansia candida (Solanaceae), Kite e Leon ${ }^{25}$ também detectaram este composto como um dos majoritários.

A influência das condições ambientais sobre o teor dos compostos voláteis característicos pode ser visualizada através da análise classificatória e de componentes principais - ACCP (Figura 1). Para

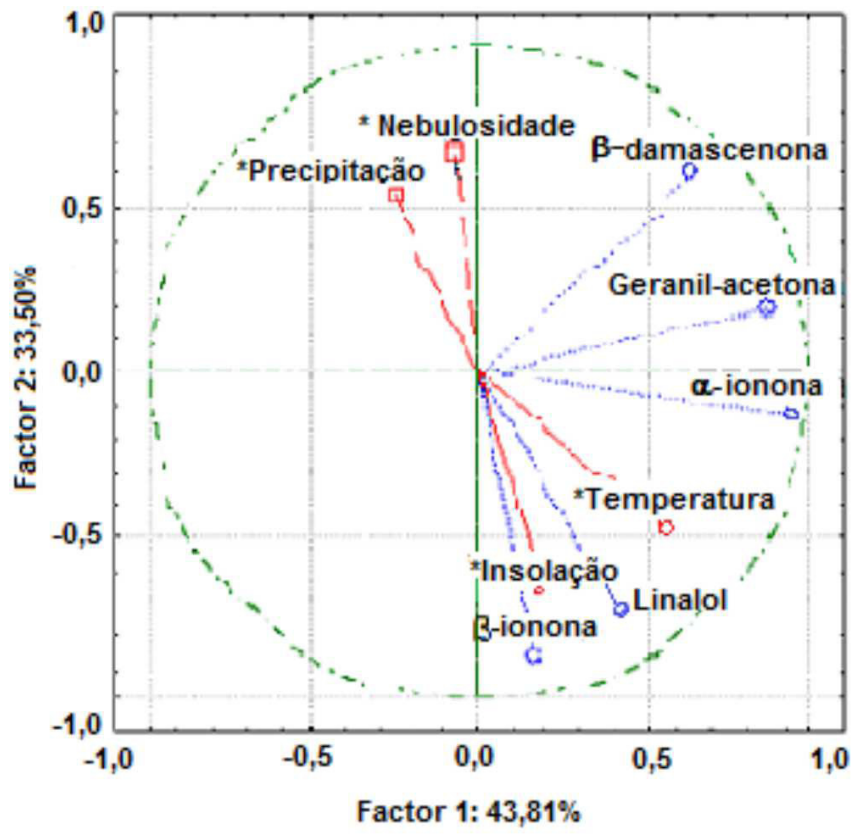

Figura 1. Análise classificatória e de componentes principais para os componentes característicos do óleo volátil foliar de D. frutescens em função das variáveis ambientais a ACCP foram utilizados os dois primeiros compostos principais, que explicam um maior percentual dos dados originais (77,31\%). Percebese que o linalol, $\alpha$-ionona e $\beta$-ionona sofrem influência diretamente proporcional da temperatura e da insolação. Além disso, pode-se dizer que estes compostos químicos sofrem uma influência inversamente proporcional das variáveis precipitação e nebulosidade. Sobre o comportamento da $\beta$-damascenona e geranil-acetona frente às condições ambientais avaliadas, nada se pode concluir pela análise realizada.

A análise de componentes principais (Figura 2) foi capaz de explicar $35,57 \%$ da variabilidade dos dados originais, com a redução de 5 variáveis iniciais, para 2 componentes principais. Por esta análise, observa-se que os óleos voláteis dos meses que compõem as estações do outono e inverno são caracterizados, principalmente, pela presença de $\beta$-damascenona e geranil-acetona em sua composição química. Já aqueles obtidos nos meses da primavera são caracterizados pela presença de linalol, $\alpha$-ionona e $\beta$-ionona em sua composição. Além disso, pode-se observar que os meses de verão têm como característica altos teores dos cinco compostos majoritários, entretanto, são caracterizados de forma mais intensa pelo linalol, $\alpha$-ionona e $\beta$-ionona.

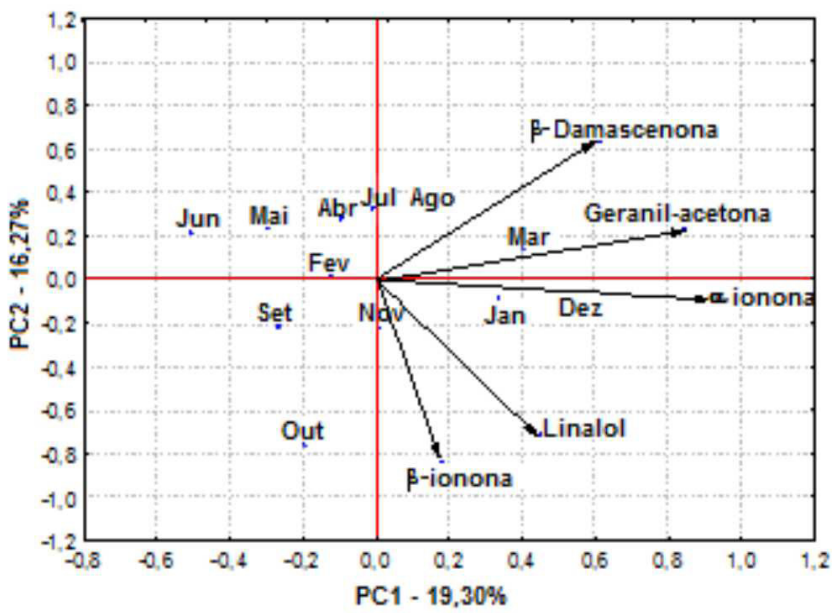

Figura 2. Análise de componentes principais para classificação dos meses em função dos constituintes químicos característicos

\section{CONCLUSÕES}

Este é o primeiro relato sobre a composição química do óleo volátil de Dalbergia frutescens e a influência das condições ambientais na espécie. Foram identificados 60 compostos voláteis, sendo detectados como compostos característicos da espécie, linalol, $\beta$-damascenona, $\alpha$-ionona, geranil-acetona e $\beta$-ionona. Observou-se que os compostos característicos são biossintetizados através da degradação de carotenoides, resultando em altos teores de $\beta$-damascenona e $\beta$-ionona.

O rendimento do óleo volátil foi fortemente influenciado pelas condições ambientais, apresentando um comportamento diretamente proporcional à temperatura e nebulosidade e inversamente proporcional à precipitação.

Em relação à influência das condições ambientais sobre a composição química, observou-se que linalol, $\alpha$-ionona e $\beta$-ionona, compostos que caracterizaram a composição química da primavera, sofreram influência diretamente proporcional da temperatura e da insolação. Observou-se ainda que as estações do outono e inverno foram caracterizadas pela ocorrência de $\beta$-damascenona e geranil-acetona compostos que, a princípio, não apresentaram relação com as condições ambientais.

Por fim, o presente estudo contribuiu para o conhecimento do potencial químico da biodiversidade local, uma vez que caracteriza parcialmente a composição química de Dalbergia frutescens. 


\section{PARTE EXPERIMENTAL}

\section{Coleta e acondicionamento do material vegetal}

As amostras de material vegetal da espécie Dalbergia frutescens foram coletadas na cidade de Chapecó-SC, sendo as coordenadas da localização: latitude $27^{\circ} 03^{\prime} 29.8^{\prime}$ 'S e longitude $52^{\circ} 39^{\prime} 36.4$ 'O. A exsicata da espécie encontra-se catalogada no herbário da Universidade Federal de Santa Maria, RS, sob o número de registro SMDB 12.936.

As amostras coletadas foram de folhas, coletando-se parcelas de material vegetal novo e velho e em todo o entorno do exemplar da espécie, visando a homogeneização das amostras. As coletas foram realizadas mensalmente de março de 2010 a fevereiro de 2011, sendo realizadas na segunda quinzena de cada mês, em horários que variaram das 14:00 às 15:00 h.

Após a coleta, as amostras foram armazenadas em freezer à $-10{ }^{\circ} \mathrm{C}$ até o momento da extração dos óleos voláteis, com o intuito de reduzir a perda dos constituintes e diminuir a atividade metabólica do material.

\section{Extração e purificação do óleo volátil}

O óleo volátil das folhas de $D$. frutescens foi obtido por hidrodestilação, utilizando-se um aparelho do tipo Clevenger modificado, com capacidade de 6 L. Para o processo de extração, o material vegetal foi previamente triturado em liquidificador, pesado e inserido no equipamento de extração, no qual o material foi mantido submerso em água destilada até o término da extração. Para este processo, fixou-se um tempo de 3 h de extração a contar do início da ebulição da água.

Após o término da extração, separaram-se os compostos voláteis da água utilizando-se como solvente extrator éter etílico bi-destilado, secou-se a mistura éter-óleo com sulfato de sódio anidro e, posteriormente, evaporou-se o solvente em banho termostático (Tecnal, modelo TE-184) a $35{ }^{\circ} \mathrm{C}$. Após a evaporação, o extrato volátil foi pesado em balança analítica (Tecnal, modelo Mark 210A classe I), para determinação do rendimento.

\section{Análise da composição química dos óleos voláteis}

\section{Análise qualitativa}

O óleo volátil foi submetido à análise em um cromatógrafo gasoso acoplado a um espectrômetro de massa, sendo a amostra $(10 \mathrm{mg} / \mathrm{mL})$ injetada no modo split (1:10). O cromatógrafo (Shimadzu, modelo GC-2010) estava equipado com coluna capilar de sílica fundida modelo RTX-5MS (Restek, $30 \mathrm{~m}$ x 0,25 mm x 0,25 $\mu \mathrm{m}$ ). As condições de análise foram: velocidade linear de $1 \mathrm{~mL} / \mathrm{min}$ com gás de arraste Hélio (He); pressão na coluna de $53 \mathrm{kPa}$; temperatura do injetor de $240{ }^{\circ} \mathrm{C}$ e temperatura do forno de 50 a $290{ }^{\circ} \mathrm{C}$, com velocidade de aquecimento de $4{ }^{\circ} \mathrm{C} / \mathrm{min}$. O espectrômetro de massa foi utilizado no modo de ionização eletrônica a $70 \mathrm{eV}$, com varredura de $\mathrm{m} / \mathrm{z}$ de 40 a $600 \mathrm{Da}$, em uma frequência de $2,94 \mathrm{scan} / \mathrm{s}$. A fonte de íons foi mantida a $260{ }^{\circ} \mathrm{C}$ e a interfase a $280{ }^{\circ} \mathrm{C}$.

Os dados obtidos foram processados utilizando o software GCMS Postrun Analysis. A identificação dos compostos foi baseada na comparação dos índices de retenção da mesma coluna e espectro de massa descrito por Mc Lafferty e Stauffer (Wiley) e por Adams. ${ }^{26} \mathrm{O}$ índice de retenção foi obtido de acordo com o método de van den Dool e Kratz para padrões de n-alcanos C7-C30 (Supelco Analytical).

\section{Análise quantitativa}

A análise quantitativa foi realizada em um cromatógrafo a gás (CG) com detector de ionização de chama (FID). O cromatógrafo (Shimadzu, modelo GC-2010) equipado com coluna capilar de sílica fundida modelo RTx-5MS (Restek, $30 \mathrm{~m}$ x 0,25 mm x 0,25 $\mu \mathrm{m}$ ) foi utilizado nas mesmas condições estabelecidas na análise qualitativa. $\mathrm{O}$ detector de ionização de chama foi mantido à temperatura de $260{ }^{\circ} \mathrm{C}$. Os dados quantitativos foram calculados pela integração da área dos picos do CG sem o uso de fatores de correção. Todas as amostras foram injetadas em triplicata.

\section{Análise estatística}

Todos os dados de rendimento e concentração dos compostos químicos identificados foram submetidos à análise de variância (ANOVA), sendo a significância das diferenças entre as médias calculadas pelo teste de Tukey, onde os valores com probabilidade inferior a 5\% foram considerados significativos.

A influência dos fatores climáticos sobre teor de compostos voláteis das folhas de $D$. frutescens foi avaliada através da análise de regressão linear múltipla, relacionando o rendimento do óleo essencial com os valores médios de temperatura, precipitação, insolação e nebulosidade da semana anterior à data da coleta, sendo que o modelo gerado foi considerado significativo para valores com probabilidade inferior a $5 \%$ e $\mathrm{R}^{2}$ ajustado superior a 0,85 . Os dados climatológicos foram disponibilizados pela Empresa de Pesquisa Agropecuária e Extensão Rural de Santa Catarina - EPAGRI em conjunto com a Estação Agrometereológica de Chapecó-SC.

A influência das condições ambientais sobre o teor dos compostos característicos de $D$. frutescens no extrato volátil foi avaliada por análise classificatória e de componentes principais (ACCP). Por fim, a análise de componentes principais (ACP) foi aplicada para examinar as relações interespecíficas entre os meses, em função dos principais constituintes químicos. Todas as análises estatísticas foram realizadas com auxílio do software Statistica 7.0.

\section{MATERIAL SUPLEMENTAR}

No material suplementar, disponível em http://quimicanova.com. br, em arquivo pdf e com acesso livre, encontram-se disponíveis cromatogramas que expressam os altos níveis de $\beta$-damascenona e $\beta$-ionona detectados nos extratos voláteis de $D$. frutescens, assim como os espectros de massa obtidos para estes compostos nas amostras analisadas.

\section{AGRADECIMENTOS}

À FAPESC pelo apoio financeiro.

\section{REFERÊNCIAS}

1. Cragg, G. M.; Newman, D. J. Em Química de produtos naturais, novos fármacos e a moderna farmacognosia; Yunes, R. A.; Cechinel-Filho, V., eds.; Ed. UNIVALI: Itajaí, 2009.

2. Henriques, A. T.; Pires, C. A. S.; Apel, M. A. Em ref. 1.

3. Souza, M. F.; Manganotti, S. A.; Souza, P. N. S.; Meira, M. R.; Matos, C. C.; Martins, E. R.; Biotemas 2011, 24, 9.

4. Stevović, S.; Ćalić-Dragosavac, D.; Mikovilović, V. S.; ZdravkovićKorać, S.; Milojević, J.; Cingel, A.; Adv. Environ. Biol. 2011, 5, 465; Taarit, M. B.; Msaada, K.; Hosni, K.; Hammami, M.; Kchouk, M. E.; Marzouk, B.; Ind. Crop Prod. 2009, 30, 333.

5. Santos, S. G.; Tese de Doutorado, Universidade Federal de Viçosa, Brasil, 2008.

6. Ribeiro, R. A.; Tese de Doutorado, Universidade Federal de Minas Gerais, Brasil, 2007.

7. Hajare, S. W.; Chandra, S.; Sharma, J.; Tandan, S. K.; Lal, J.; Telang, A. G.; Fitoterapia 2001, 72, 131; Kale, M.; Misar, A. V.; Dave, V.; 
Joshi, M.; Mujumdar, A. M.; J. Ethnopharmacol. 2007, 112, 300; Yu, X.; Wang, W.; Yang, M.; Food Chem. 2007, 104, 715; Wang, W.; Weng, X.; Cheng, D.; Food Chem. 2000, 71, 45; Songsiang, U.; Wanich, S.; Pitchuanchom, S.; Netsopa, S.; Uanporn, K.; Yenjai, C.; Fitoterapia 2009, 80, 427 .

8. Khan, I. A.; Avery, M. A.; Burandt, C. L.; Goins, D. K.; Mikell, J. R.; Nash, T. E.; Azadegan, A.; Walker, L. A.; J. Nat. Prod. 2000, 63, 1414.

9. Carvalho, A. M.; Brittonia 1997, 49, 87.

10. Ribeiro, R. V.; Machado, E. C.; Oliveira, R. F.; Ciênc. Agrotec. 2006, 30,670 .

11. Taiz, L.; Zeiger, E.; Fisiologia Vegetal, $3^{\text {a }}$ ed., Artmed: Porto Alegre, 2006.

12. Fitter, A. H.; Hay, R. K. M.; Environmental physiology of plants, $3^{\text {rd }}$ ed., Academic Press: London, 2002.

13. Vitti, A. M. S.; Brito, J. O.; Sci. For. 1999, 56, 145.

14. Petropoulos, S. A.; Daferera, D.; Polissiou, M. G.; Passam, H. C.; Sci. Hortic. 2008, 115, 393.

15. Zehtab-Salmasi, S.; Javanshir, A.; Omidbaigi, R.; Alyari, H.; GhassemiGolezani, K.; Acta Agron. Hung. 2001, 49, 75.

16. Manochai, B.; Paisooksantivatana, Y.; Choi, H.; Hong, J. H.; Sci. Hortic. 2010, 126, 462 .
17. Baldermann, S.; Kato, M.; Kurosawa, M.; Kurobayashi, Y.; Fujita, A.; Fleischmann, P.; Watanabe, N.; J. Exp. Bot. 2010, 61, 2967.

18. Simkin, A. J.; Underwood, B. A.; Auldridge, M.; Loucas, H. M.; Shibuya, K.; Schmelz, E.; Clark, D. G.; Klee, H. J.; Plant Physiol. 2004, 136,3504

19. Yamamizo, C.; Kishimoto, S.; Ohmiya, A.; J. Exp. Bot. 2010, 61, 709.

20. Hatanaka, A.; Phytochemistry 1993, 34, 1201.

21. Rezende, C. M.; Corrêa, V. F. S.; Costa, A. V. M.; Castro, B. C. S.; Quim. Nova 2004, 27, 414.

22. Fernandes, J. M.; Dissertação de Mestrado, Universidade Federal de Viçosa, Brasil, 2007.

23. Malerbo-Souza, D. T.; Nogueira-Couto, R. H.; Couto, L. A.; Souza, J. C.; Braz. J. Vet. Res. Anim. Sci. 2003, 40, 272.

24. Pham-Delegue, M. H.; Etievant, P.; Guichard, E.; Masson, C.; J. Chem. Ecol. 1989, 15, 329

25. Kite, G. C.; Leon, C.; Phytochemistry 1995, 40, 1093.

26. Adams, R.; Essential oil components by Quadrupole GC/MS, Allured Publishing Corp.: Carol Stream, 2001. 


\section{EFEITOS DAS CONDIÇÕES AMBIENTAIS SOBRE O TEOR E VARIABILIDADE DOS ÓLEOS VOLÁTEIS DE}

Dalbergia frutescens (Vell.) Britton (Fabaceae)

\section{Caroline Eliza Mendes*, Fabiana Casarin e Anderson Luis Ohland}

Área de Ciências Exatas e Ambientais, Universidade Comunitária da Região de Chapecó, Av. Senador Atílio Fontana, 591E, 89809-000 Chapecó - SC, Brasil

\section{Adriana Flach e Luiz Antonio Mendonça Alves da Costa}

Departamento de Química, Universidade Federal de Roraima, Av. Cap. Enê Garcêz, 2413, B1. III, 69304-000 Boa Vista - RR, Brasil Rosiane Berenice Nicoloso Denardin

Curso de Agronomia, Universidade Federal da Fronteira Sul, Av. Pres. Getúlio Vargas, 609N, 89812-000 Chapecó - SC, Brasil Neusa Fernandes de Moura

Escola de Química e Alimentos, Universidade Federal do Rio Grande, Rua Barão do Caí, 125, 955000-000 Santo Antonio da Patrulha - RS, Brasil

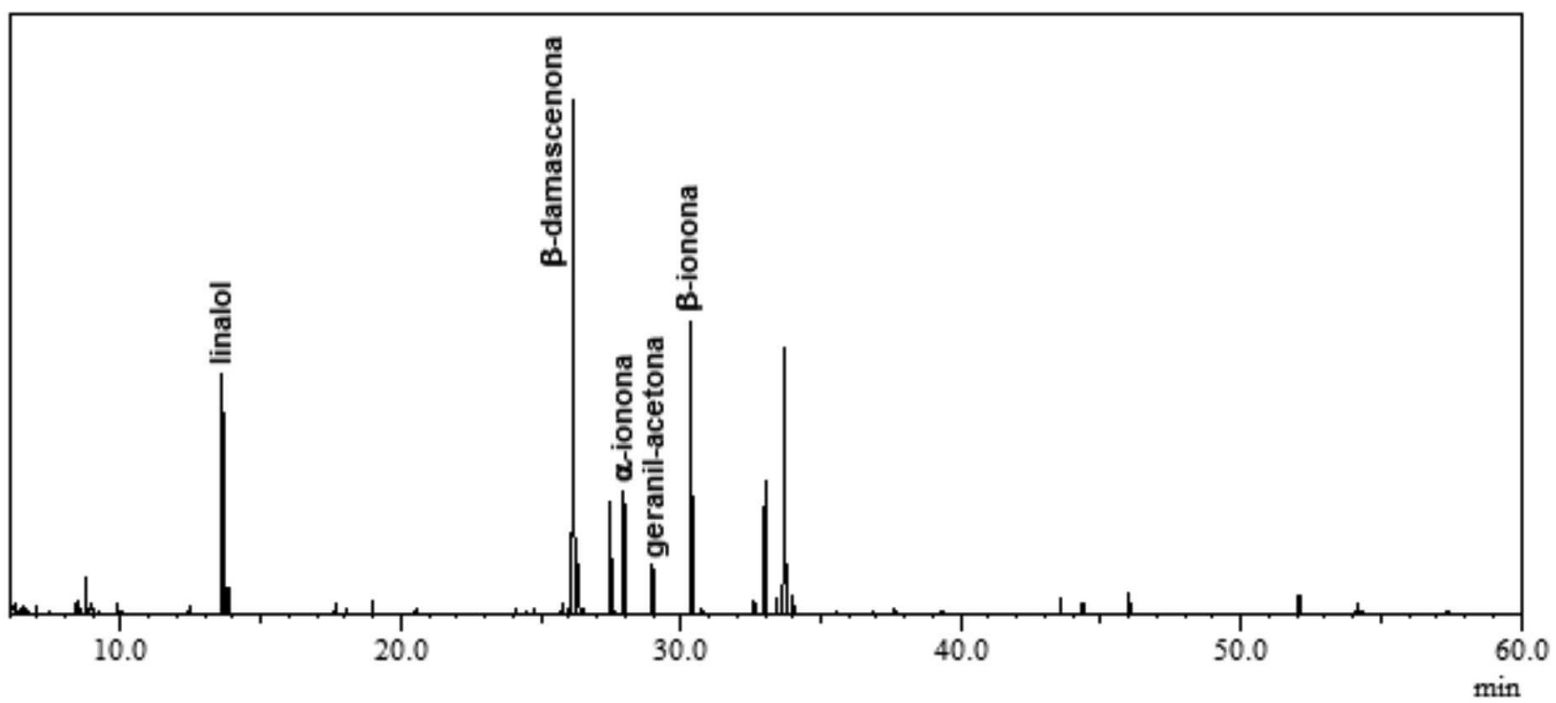

Figura 1S. Cromatograma para a amostra de março de 2010 dos compostos voláteis detectados de D. frutescens, ressaltando a máxima concentração de $\beta$-damascenona obtida durante o período analisado

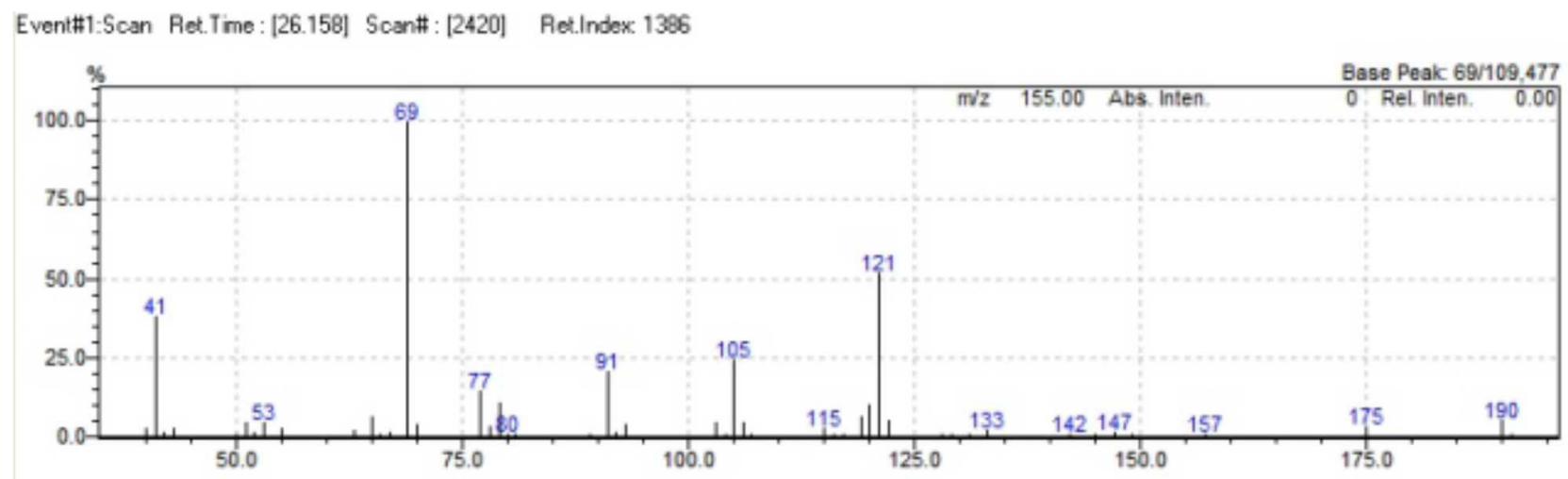

Figura 2S. Espectro de massas da $\beta$-damascenona obtida através do software GC-Solution dos extratos voláteis de D. frutescens 


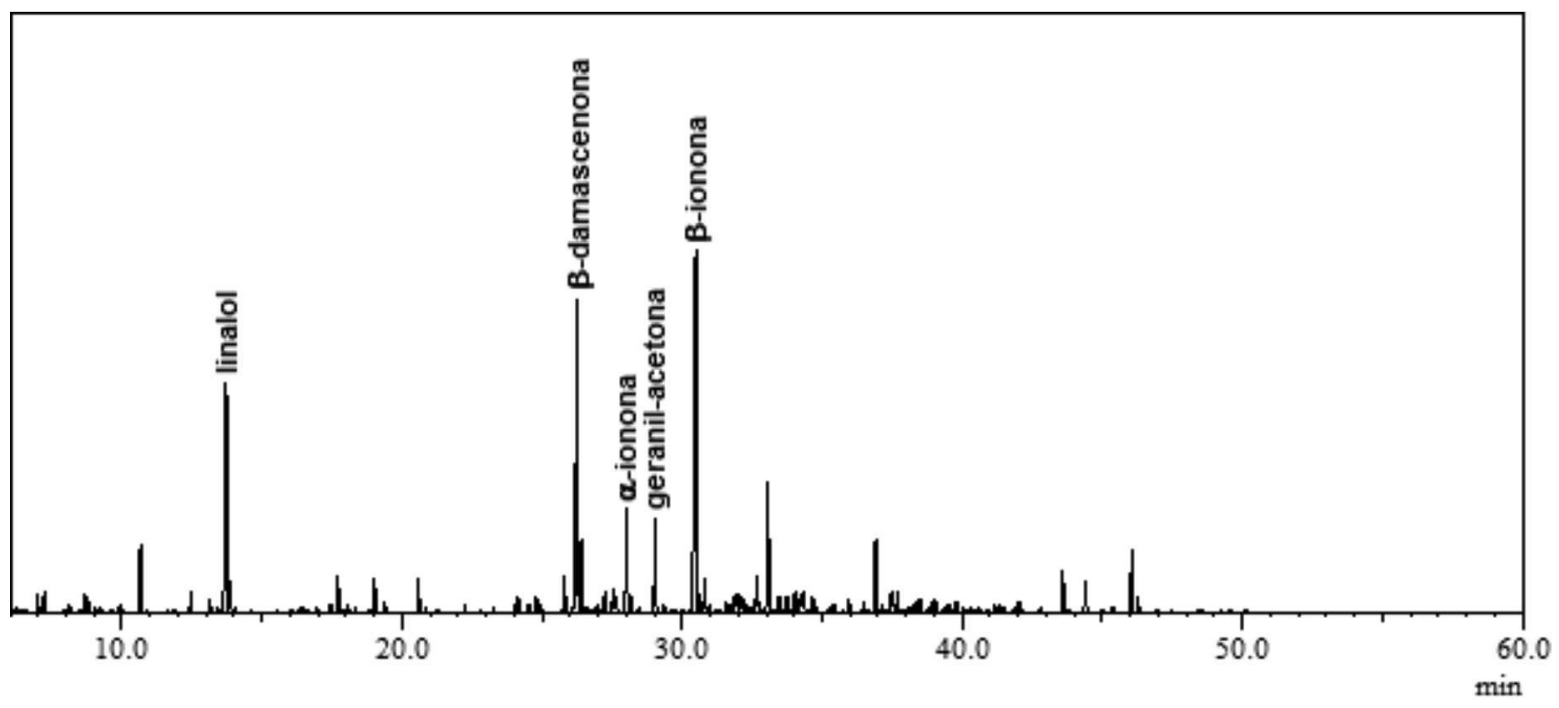

Figura 3S. Cromatograma para a amostra de dezembro de 2010 dos compostos voláteis detectados de D. frutescens, ressaltando a máxima concentração de $\beta$-ionona obtida durante o período analisado

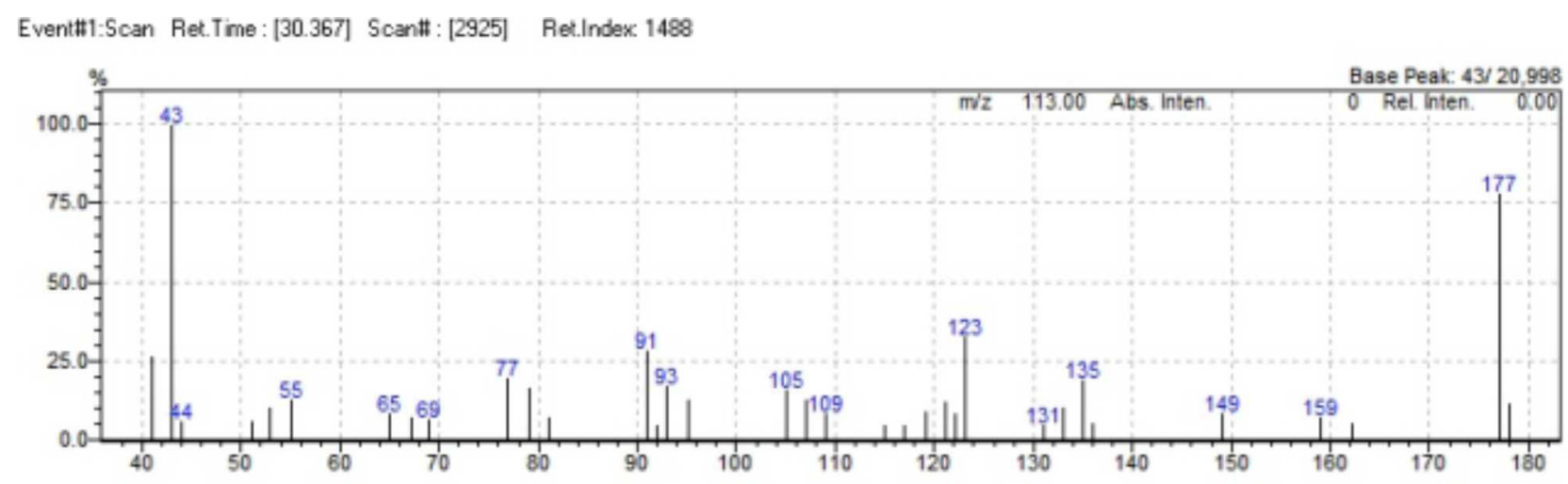

Figura 4S. Espectro de massas da $\beta$-ionona obtida através do software GC-Solution dos extratos voláteis de D. frutescens 